\title{
Plantas medicinais utilizadas na caatinga brasileira e o potencial terapêutico dos
} metabólitos secundários: uma revisão

\author{
Medicinal plants used in the Brazilian caatinga and the therapeutic potential of secondary \\ metabolites: a review
}

Plantas medicinales utilizadas en la caatinga brasileña y el potencial terapéutico de metabolitos secundarios: una revisión

Recebido: 26/09/2021 | Revisado: 02/10/2021 | Aceito: 04/10/2021 | Publicado: 09/10/2021

Geovan Figueirêdo de Sá-Filho ORCID: https://orcid.org/0000-0003-0380-1906 Universidade Federal do Rio Grande do Norte, Brasil E-mail: geovan.sa@hotmail.com

Antonia Isabelly Bezerra da Silva ORCID: https://orcid.org/0000-0002-3718-470X Universidade Estadual do Rio Grande do Norte, Brasil E-mail: antoniaisabelly@alu.uern.br

Elanny Mirelle da Costa

ORCID: https://orcid.org/0000-0002-2947-7529 Universidade Estadual do Rio Grande do Norte, Brasil E-mail: elannymirelle@gmail.com Luanne Eugênia Nunes

ORCID: https://orcid.org/0000-0001-6524-0994 Faculdade Nova Esperança de Mossoró, Brasil E-mail: luanneeugenia@ @acenemossoro.com.br

Louise Helena de Freitas Ribeiro ORCID: https://orcid.org/0000-0002-8729-013X Faculdade Nova Esperança de Mossoró, Brasil E-mail: louise@ facenemossoro.com.br

José Rodolfo Lopes de Paiva Cavalcanti

ORCID: https://orcid.org/0000-0002-1554-3249 Universidade Estadual do Rio Grande do Norte, Brasil E-mail: rodolfolopes@uern.br

Fausto Pierdona Guzen

ORCID: https://orcid.org/0000-0002-5458-7236 Universidade Estadual do Rio Grande do Norte, Brasil E-mail: faustoguzen@uern.br

Lucídio Clebeson de Oliveira

ORCID: https://orcid.org/0000-0002-2033-7546 Universidade Estadual do Rio Grande do Norte, Brasil

E-mail: lucidioclebeson@uern.br

Jeferson de Souza Cavalcante

ORCID: https://orcid.org/0000-0002-2714-0746 Universidade Federal do Rio Grande do Norte, Brasil E-mail: jeferson.cavalcante@ufrn.br

\begin{abstract}
Resumo
O bioma Caatinga possui uma ampla diversidade e proporciona a população a utilização de plantas para fins medicinais. A indústria farmacêutica tem um grande interesse em pesquisar os metabólitos presentes nas espécies desse bioma, além de comprovar cientificamente qual patologia pode ser tratada utilizando a espécie em estudo. Muitas espécies que são utilizadas pela população ainda não foram estudadas. Esse estudo teve como objetivo buscar informações acerca das plantas medicinais da Caatinga, reunir as informações históricas sobre as espécies utilizadas pela população, abordar os principais métodos de extração e as classes de metabólitos secundários com suas respectivas propriedades medicinais. Para elaboração desta revisão integrativa foram feitas pesquisas a partir de bancos de dados como SCIELO, LILACS, PUBMED e Science direct. Os artigos foram pesquisados em língua portuguesa e inglesa, tendo sido a maioria publicada nos últimos 20 anos. Diversas famílias de plantas foram descritas nos estudos, porém a família com maior quantidade de espécie que possui potencial medicinal foi a família Fabaceae. A forma de preparação da planta para o uso varia de acordo com a tradição cultural local, mas a que predomina é a infusão e a maceração.
\end{abstract}

Palavras-chave: Espécies medicinais; Fitoquímica; Fitoterapia; Semiárido. 


\begin{abstract}
The Caatinga biome has a wide diversity and provides the population with the use of plants for medicinal purposes. The pharmaceutical industry has a great interest in researching the metabolites present in the species of this biome, in addition to scientifically proving which pathology can be treated using the species under study. Many species that are used by the population have not yet been studied. This study aimed to seek information about medicinal plants in the Caatinga, gather historical information about the species used by the population, address the main extraction methods and the classes of secondary metabolites with their respective medicinal properties. For the elaboration of this integrative review, searches were carried out from databases such as SCIELO, LILACS, PUBMED and Science direct. The articles were searched in Portuguese and English, with the majority having been published in the last 20 years. Several plant families have been described in the studies, but the family with the largest amount of species that has medicinal potential was the Fabaceae family. The way in which the plant is prepared for use varies according to the local cultural tradition, but the predominant one is infusion and maceration.
\end{abstract}

Keywords: Medicinal species; Phytochemistry; Phytotherapy; Semiarid.

\title{
Resumen
}

El bioma de Caatinga tiene una amplia diversidad y proporciona a la población el uso de plantas con fines medicinales. La industria farmacéutica tiene un gran interés en investigar los metabolitos presentes en las especies de este bioma, además de demostrar científicamente qué patología se puede tratar utilizando la especie en estudio. Muchas especies que son utilizadas por la población aún no han sido estudiadas. Este estudio tuvo como objetivo buscar información sobre plantas medicinales en la Caatinga, recabar información histórica sobre las especies utilizadas por la población, abordar los principales métodos de extracción y clases de metabolitos secundarios con sus respectivas propiedades medicinales. For the elaboration of this integrative review, searches were carried out from databases such as SCIELO, LILACS, PUBMED and Science direct. The articles were searched in Portuguese and English, with the majority having been published in the last 20 years. En los estudios se describieron varias familias de plantas, pero la familia con mayor cantidad de especies que tiene potencial medicinal fue la familia Fabaceae. La forma de preparación de la planta para su uso varía según la tradición cultural local, pero la predominante es la infusión y la maceración.

Palabras clave: Especies medicinales; Fitoquímica; Fitoterapia; Semi árido.

\section{Introdução}

A biodiversidade de plantas do Brasil é considerada a maior do mundo, são mais de 55 mil espécies descritas, correspondente a $22 \%$ do número total de espécies do planeta (Carvalho et al., 2007). Em média, 40\% do planeta é formado com florestas tropicais e subtropicais e $42 \%$ destas formam as florestas secas, onde está inclusa a caatinga (Moreira et al., 2006).

A Caatinga, um bioma exclusivamente brasileiro e com ampla diversidade vegetal e animal (Guedes et al., 2014), tem proporcionado a população local a utilização de plantas com finalidade medicinal (Gomes et al., 2008). Em decorrência disso a indústria farmacêutica despertou um interesse crescente pelas pesquisas de plantas medicinais com potencial terapêutico neste bioma. Os estudos etnobotânicos que têm sido realizados na região mostram que as plantas da Caatinga são utilizadas pela comunidade para tratar doenças variadas, como doenças de pele, doenças respiratórias e gastrintestinais, além de infecções variadas (Trovão et al., 2007).

Esse interesse em pesquisar as propriedades medicinais das plantas da Caatinga, para fins terapêuticos, vêm sendo exploradas extensivamente pelos pesquisadores e pela indústria farmacêutica, interessada em desenvolver novos medicamentos. Isso se deve, sobretudo, à grande tradição do uso das plantas pela medicina popular (Calixto \& Siqueira, 2008), já que o uso tradicional de plantas pela população é feito sem o conhecimento científico, apenas levando em conta as informações terapêuticas que foram sendo acumuladas durante séculos (Maciel et al., 2002). As análises científicas dessas espécies de plantas da Caatinga podem possibilitar um melhor entendimento de quais propriedades, e uma consequente dosagem, corresponde a um efeito terapêutico na população.

Apesar do número de estudos em relação ao potencial biológico de plantas da Caatinga ter crescido (Gomes et al., 2008), muitas plantas que são utilizadas pelas comunidades para fins medicinais ainda não foram submetidas a estudos científicos para confirmar sua eficácia no tratamento de doenças (Silva et al., 2015). 
Os estudos dos constituintes presentes nos extratos botânicos, extratos esses obtidos através de processos de extração já amplamente conhecidos pela ciência e população, são necessários para embasar o inicio da investigação científica, principalmente em relação aos metabólitos secundários que geralmente são os responsáveis por ações biológicas ligados aos extratos (Sonaglio et al., 2010). Outro parâmetro que influencia na extração desses metabólitos secundários das plantas medicinais é o tipo de solvente utilizado, isso terá um intima relação com o sucesso da abordagem terapêutica. Além disso, existem outros parâmetros básicos que influenciam na qualidade de um extrato como a metodologia de extração e a parte da planta utilizada (Ncube et al., 2011).

Neste sentido, o estudo objetiva reunir informações sobre as principais espécies e famílias de plantas medicinais da caatinga, relatando um contexto histórico de uso pela população da região além de esclarecer os principais métodos extrativos e as classes de metabólitos secundários e suas respectivas propriedades medicinais de plantas utilizadas pela população da região semiárida.

\section{Metodologia}

A revisão integrativa é um método que proporciona a síntese de conhecimento e a incorporação da aplicabilidade de resultados de estudos significativos na prática (Koche, 2011; Ludke \& Andre, 2013; Estrela, 2018). Nesse sentido, o presente trabalho trata-se de uma revisão integrativa realizada por meio de levantamentos bibliográficos presentes em bases de dados científicas (Pereira et al., 2018). Para elaboração desta revisão integrativa foram feitas pesquisas a partir de bancos de dados como SCIELO, LILACS, PUBMED e Science direct (Tabela 1). Os artigos foram pesquisados em língua portuguesa e inglesa, tendo sido a maioria publicada nos últimos 20 anos. Os artigos foram selecionados inicialmente pelos títulos, e a seleção foi refinada pelos resumos. Após a leitura dos resumos, os estudos que pareceram corresponder aos objetivos dessa revisão foram lidos integralmente e participaram deste estudo.

As palavras utilizadas na busca de informações deste artigo foram: "Caatinga", "Plantas Medicinais", "Propriedades Terapêuticas" e "Metabólitos Secundários". Os dados presentes nesta revisão foram encontrados nos próprios artigos sobre os temas pesquisados. Os critérios de inclusão para seleção de artigos foram estudos que descrevessem diversas espécies de plantas medicinais utilizadas pela população tradicional da Caatinga, além das formas de obtenção dos extratos botânicos e da identificação dos metabólitos secundários. Quanto aos critérios de exclusão, foram desconsiderados os artigos que não trouxessem relevância empírica para o processo de evolução do conhecimento sobre as plantas medicinas pela população da Caatinga.

Tabela 1. Lista de banco de dados e a quantidade de artigos selecionados após critérios de inclusão e exclusão.

Bancos de dados

\begin{tabular}{|c|c|}
\hline SCIELO & 21 \\
\hline LILACS & 12 \\
\hline PUBMED & 7 \\
\hline Science direct & 4 \\
\hline TOTAL & 44 \\
\hline
\end{tabular}

Fonte: Autores. 


\section{Resultados e Discussão}

\subsection{Histórico do uso de plantas medicinais na história humana com enfoque no bioma caatinga}

Desde os tempos remotos que o homem busca recursos na natureza para melhorar sua qualidade de vida e aumentar sua chance de sobreviver (Lorenzi e Matos, 2008). As plantas medicinais são os mais antigos meios empregados pelo homem no tratamento de enfermidades de todos os tipos, isto é, a utilização de plantas na prevenção e/ou na cura de doenças é uma prática que sempre existiu na história da humanidade (Moraes \& Santana, 2001).

Existem registros da utilização de plantas no tratamento das enfermidades desde 4.000 a.C (Helfand \& Cowen, 1990). Em meio às tentativas e erros o homem primitivo passou a adquirir conhecimentos sobre as plantas, identificando quais poderiam ser utilizadas como alimentos, medicamentos e determinando aquelas que poderiam apresentar algum perigo a saúde, como as plantas venenosas (Amorozo et al., 1996).

A natureza oferece ao homem uma grande quantidade de plantas com valores medicinais. Um conhecimento que antes era baseado apenas em crenças, hoje tem bases científicas que comprovam a sua eficácia (Yadav, 2011). Atualmente, a dificuldade de assistência médica pela população, o alto custo dos medicamentos industrializados, aliado à tendência da utilização de produtos de origem natural contribuem para aumentar o uso das plantas como recurso terapêutico (Badke et al., 2012). Além disso, o uso de plantas medicinais é conhecido e aplicado em diversas culturas de todo o mundo e em variadas formas. Isso mostra sua importância, principalmente, em lugares que o serviço de saúde não é muito acessível (Agra et al., 2008).

O conhecimento popular relacionado às plantas medicinais é base para a medicina popular no Brasil, é derivado de culturas indígenas brasileiras com influência dos europeus e africanos no período da colonização (Martins et al., 2000). Segundo a Organização Mundial de Saúde (OMS), 80\% da população de países desenvolvidos utilizam práticas tradicionais na atenção primária à saúde, sendo que $85 \%$ dessa população fazem uso de plantas medicinais (Rosa et al., 2011). Calixto (2001) afirma que cerca de $40 \%$ dos medicamentos disponíveis foram desenvolvidos a partir de fontes naturais, sendo que $25 \%$ de plantas, $12 \%$ de microrganismos e $3 \%$ de animais.

No Brasil, o Ministério da Saúde aprovou em 2006, pela portaria n 648, a Política Nacional de Atenção Básica que inclui as plantas medicinais no SUS e pelo Decreto n 5.813 de 2006, a Política Nacional de Plantas Medicinais e Fitoterápicos. Esta última estabelece diretrizes e linhas prioritárias para o desenvolvimento de ações pelos diversos parceiros em torno de objetivos comuns voltados à garantia de acesso seguro e uso racional de plantas medicinais e fitoterápicos em nosso país, ao desenvolvimento de tecnologias e inovações, assim como ao fortalecimento das cadeias e dos arranjos produtivos, ao uso sustentável da biodiversidade brasileira e ao desenvolvimento do complexo produtivo da saúde (Brasil, 2006).

A biodiversidade da vegetação do Brasil é uma das maiores a nível mundial, existem mais de 55 mil espécies descritas no território nacional, ou seja, $22 \%$ do número total de espécies do planeta. Em média $40 \%$ do planeta é formado com florestas tropicais e subtropicais e 42\% destas formam as florestas secas, onde está inclusa a caatinga (Moreira et al., 2006).

A caatinga é um bioma que possui $844.000 \mathrm{Km}^{2}$, o que corresponde a $10 \%$ da área do país e tem uma população de mais de 23 milhões de pessoas (MMA, 2010). Envolve áreas dos Estados do Ceará, Rio Grande do Norte, Paraíba, Pernambuco, Alagoas, Sergipe, o sudoeste do Piauí, partes do interior da Bahia e do norte de Minas Gerais (Figura 1; Andrade et al., 2005). 
Figura 1. Destaque do território brasileiro com a representação do bioma Caatinga, e as abrangência territorial nos diferentes estados do Brasil.

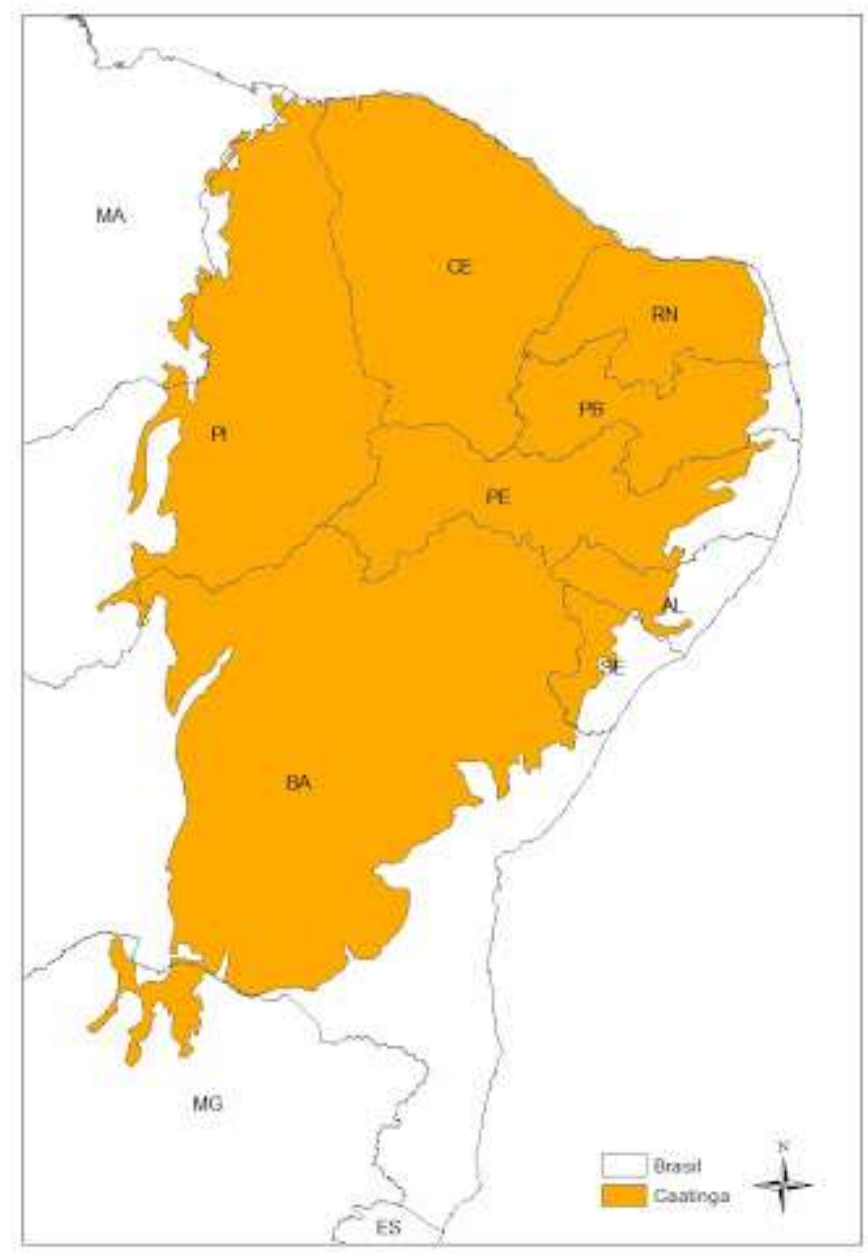

Fonte: SNIF FLORESTAL (2021).

Historicamente, a Caatinga teria sido negligenciada por conta das suas características visuais onde acreditavam que se tratava de um bioma homogêneo, com baixa riqueza e endemismos (Vanzolini, 1976). Outros estudos têm mostrado que a Caatinga possui um bioma bastante heterogêneo (Guedes et al., 2014), com clima quente e chuva distribuída no período de três a seis meses durante o ano (Velloso et al., 2002). A região é altamente impactada por atividades de agropecuária e extrativismo (Giulietti et al., 2004), o que tem levado a uma formação de desertificação, e como consequência, a uma perda de diversidade que ainda não foi mensurada (Camardelli e Napoli, 2012). Apesar de ser o único bioma exclusivamente brasileiro, apenas 1\% do seu território é protegido por unidades de conservação de proteção integral (Leal et al., 2005), tornado o bioma brasileiro menos protegido (MMA, 2004).

Além de o bioma estar ameaçado, a investigação sobre o potencial de sua vegetação se torna imprescindível para que haja um melhor planejamento e ocorra a sua conservação e recuperação (Santana e Souto, 2006). O Ministério do Meio Ambiente afirma que a Caatinga apresenta elevada heterogeneidade vegetacional, caracterizada por distintas fisionomias e elevado endemismo, fazendo deste um ambiente de extrema importância biológica (MMA, 2003). Umas das finalidades de se investigar as espécies oriundas da Caatinga é o uso medicinal de suas plantas, visto que seu potencial terapêutico é conhecido há décadas (Agra et al., 2007). As espécies possuem características fisiológicas com adaptações complexas e peculiares às 
condições do ambiente onde vivem e tem despertado o interesse dos cientistas. Os estudos etnobotânicos que têm sido realizados na região mostram que as plantas da Caatinga são utilizadas pela comunidade para tratar doenças variadas, como doenças de pele, doenças respiratórias e gastrintestinais, além de infecções variadas (Trovão et al., 2007).

Apesar do número de estudos em relação ao potencial biológico de plantas da Caatinga ter crescido, muitas plantas que são utilizadas pelas comunidades para fins medicinais ainda não foram submetidas a estudos científicos para confirmar sua eficácia no tratamento de algumas doenças (Silva et al., 2015; Silva et al., 2021). Dados fitoquímicos e farmacológicos dessas espécies precisam ser analisados para avaliar se o uso medicinal das mesmas corresponde aos metabólitos presentes (Cartaxo et $a l, 2010)$.

\subsection{Espécies de plantas utilizadas pela população da caatinga e seu potencial terapêutico}

Atualmente, aproximadamente $48 \%$ dos medicamentos utilizados na terapêutica é resultado de forma direta ou indireta da exploração de produtos naturais, principalmente das plantas medicinais através do conhecimento etnofarmacológico (Carvalho et al., 2007). Na caatinga nordestina estas plantas são amplamente utilizadas na medicina popular pelas comunidades locais. Estas comunidades possuem uma vasta farmacopeia natural, em boa parte proveniente dos recursos vegetais encontrados nos ambientes naturais ocupados por estas populações, ou cultivados em ambientes antropicamente alterados. Diante desse quadro, é possível constatar o grande interesse por plantas medicinais, tanto em âmbito nacional quanto internacional, pois estas apresentam um potencial terapêutico e econômico, visado especialmente pela indústria farmacêutica que realiza a prospecção de novos produtos (Morgan, 1995; Albuquerque, 1997). Conhecer as possibilidades de usos, locais de aquisição e as partes das plantas medicinais utilizadas, é muito importante para a correta utilização das espécies de plantas medicinais presente na Caatinga.

Alguns estudos se propuseram a analisar as plantas medicinais e as finalidades terapêuticas das espécies utilizadas pela população da Caatinga tradicionalmente, mesmo com diferentes objetivos é possível perceber um crescente conhecimento científico sobre a utilização de plantas medicinais neste Bioma (Fernandes e Bezerra, 2020; Castro et al., 2021; Cruz et al., 2021).

Diversas espécies de plantas foram catalogadas com finalidades medicinais distribuídas em diferentes famílias e gêneros. Dentre as famílias de plantas relatadas com potencial medicinal a que apresentou maior quantidade de espécies foi a ; família Fabaceae (Tabela 2). Sabendo que a família Fabaceae constitui uma das famílias botânicas de maior importância econômica e medicinal (Gomes et al., 2008), as espécies relatadas possibilitam levantar as principais propriedades curativas e terapêuticas relacionadas as plantas medicinais utilizadas em ambiente de Caatinga. Muitas das plantas descritas são usadas tanto na farmacologia quanto na medicina popular, essa abordagem da medicina popular já é mencionada em estudos com comunidades rurais da Caatinga, enfatizando a influência desse grupo de plantas na cultura popular nordestina (Loiola et al., 2010).

Tabela 2. Levantamento das famílias/nomes científicos, nomes populares e os principais usos terapêuticos das plantas medicinais pela população Nordestina.

\begin{tabular}{lll}
\hline Família/Nome científico & Nome popular & Uso terapêutico \\
\hline Anacardiaceae & & \\
Anacardium ocidentalis $L$. & Cajueiro & Gripe, inflamação \\
Myracrotrum urundeuva Allemão & Aroeira & Inflamação \\
Annonaceae & & \\
Annona sp. & Imbiriba & Amidalite
\end{tabular}




\begin{tabular}{|c|c|c|}
\hline Xylopia aromatica (Lam.) Mart. & Pimenta de macaco & Dores \\
\hline Xylopia serica St Hill & Araticum & Próstata \\
\hline \multicolumn{3}{|l|}{ Asteraceae } \\
\hline Aconthospermum sp. & Capim de caboclo & Sinusite, gripe \\
\hline Baccharis sylvestris $L$. & Alecrim & Aneurisma, gripe, febre \\
\hline Martricaria chamomilla $L$. & Camomila & Nervos \\
\hline Egletes viscosa (L.) Less. & Marcela & Problemas estomacais \\
\hline Helianthus annus L. & Girassol & Dores \\
\hline \multicolumn{3}{|l|}{ Bignoniaceae } \\
\hline Anemopaegma arvenses (Vell.) & Catuaba & Impotência sexual \\
\hline Tabebuia caraiba (Mart.) Ber. & Craibeira & Analgésico \\
\hline \multicolumn{3}{|l|}{ Bombacaceae } \\
\hline Bombax coreaceae Mart. & Umburuçú & Hérnia, coluna \\
\hline \multicolumn{3}{|l|}{ Cacteceae } \\
\hline Melocactus sp. & Raiz de quipá & Catarro preso \\
\hline \multicolumn{3}{|l|}{ Capifloraceae } \\
\hline Sambucus nigra $L$. & Flor de sabugueiro & Dentição \\
\hline \multicolumn{3}{|l|}{ Cecropiaceae } \\
\hline Cecropia sp. & Umburana de cheiro & Problemas estomacais, gripe, pressão alta \\
\hline \multicolumn{3}{|l|}{ Convolvulaceae } \\
\hline Ipomoea purga (Wender.) Hayne & Batata de purga & Laxante, gripe, vermes \\
\hline \multicolumn{3}{|l|}{ Cucurbitaceae } \\
\hline Luffa operculata (L.) Cogn. & Cabacinha & Sinusite, abortivo \\
\hline Wilbrandia sp. & Batata de teiú & Próstata, rins \\
\hline \multicolumn{3}{|l|}{ Euphorbiacea } \\
\hline Cnidoscolus phyllacanthus Pax \& Hoffm. & Favela & Intestino, fígado \\
\hline Croton rhamniflolius H.B.K. & Quebra fraco & Fraqueza, fígado, estimulante do apetite \\
\hline Euphorbia phosphorea Mat. & Pau de leite & Infertilidade feminina, inflamação \\
\hline \multicolumn{3}{|l|}{ Krameriaceae } \\
\hline Krameria tomentosa A. St. Hil. & Carqueja & Colesterol \\
\hline \multicolumn{3}{|l|}{ Lamiaceae/Labiatae } \\
\hline Plectranthus barbatus Andrews & Boldo & Problemas estomacais \\
\hline Rosmaris officinalis $L$. & Alecrin de caboclo & Gripe \\
\hline \multicolumn{3}{|l|}{ Lauraceae } \\
\hline Cinnamomum zeylanicum Beryn. & Canela & Nervos, sangue \\
\hline \multicolumn{3}{|l|}{ Fabaceae } \\
\hline Anadenthera peregrina (L.) Speg. & Angico & Gripe, expectorante \\
\hline Bauhinia forficata Link. & Mororó & Diabetes, colesterol \\
\hline Bowdicha major Benth. & Sucupira & Coluna, reumatismo, dor de cabeça, aneurisma \\
\hline Caesalpinea ferrea Mart. & Pau ferro & Gripe, asma \\
\hline Caesalpinea piramdalys Tul. & Catingueira & Útero, ovário, próstata \\
\hline Chamaecrista ensiformis (Vell) & Pau ferro & Gripe, asma, anemia \\
\hline Erytrina mulungu Mart. Ex. Benth & Mulungu & Nervos, insônia, pressão atla, dores de cabeça \\
\hline Leucaena leucocephala Lam. & Leucena & Nervos, problemas estomacais \\
\hline
\end{tabular}




\begin{tabular}{|c|c|c|}
\hline Mimosa sp. & Jurema preta & Cicatrizante \\
\hline Stryphnodendron barbatimao Mart. & Barbatimão & Inflamação; gastrite \\
\hline \multicolumn{3}{|l|}{ Linaceae } \\
\hline Linum usitaissimum L. & Linhaça & Colesterol \\
\hline Illicium verum Hook. F. & Anis estrelado & Dor de cabeça \\
\hline \multicolumn{3}{|l|}{ Myristicaceae } \\
\hline Myristica frangans Houtt. & Noz-moscada & Dor de cabeça \\
\hline \multicolumn{3}{|l|}{ Myrtaceae } \\
\hline Eucaliptus sp. & Eucalipto & Gripe, expectorante \\
\hline \multicolumn{3}{|l|}{ Olacaceae } \\
\hline Olea europaea $L$. & Oliveira & Todos os males \\
\hline Ximenia americana $L$. & Ameixa & Inflamação, dores \\
\hline \multicolumn{3}{|l|}{ Phytolacaceae } \\
\hline Petiveria alliceae $L$. & Tipi, tipé & Reumatismo \\
\hline \multicolumn{3}{|l|}{ PoaceaeA } \\
\hline Cymbopogon citratus (DC.) Stapf. & Pichuri & Dores \\
\hline \multicolumn{3}{|l|}{ Punicaceae } \\
\hline Punica granatum L. & Romã & Amidalite \\
\hline \multicolumn{3}{|l|}{ Rubiaceae } \\
\hline Cephaelus ipecacunha Rich. & Papaconha & Gripe, ameba \\
\hline Coutarea hexandra Schum & Quina-quina & Febre, sinusite, dor de cabeça, fraqueza \\
\hline \multicolumn{3}{|l|}{ Sapotaceae } \\
\hline Bumelia sertorum Mart. & Quixabeira & Rins; inflamação \\
\hline \multicolumn{3}{|l|}{ Terstroemiaceae } \\
\hline Thea sinensis $L$. & Chá preto & emagrecimento \\
\hline \multicolumn{3}{|l|}{ Tiliaceae } \\
\hline Triumpheta semitrilobata $L$. & Carrapicho de boi & fluxo menstrual \\
\hline \multicolumn{3}{|l|}{ Umbeliferae } \\
\hline Foeniculum vulgare Mill. & Endro & Dentição \\
\hline Pimpinella anisum L. & Erva doce & Dentição \\
\hline \multicolumn{3}{|l|}{ Zingiberaceae } \\
\hline Zingiber officinalis $L$. & Gengibre & Gripe \\
\hline
\end{tabular}

As principais atividades terapêuticas descrita nos estudos envolvendo as espécies de plantas da família Fabaceae relatam, além dos efeitos anti-inflamatórios, alguns efeitos anti-helmínticos; antinociceptivos, antiepiléticos, antioxidantes, analgésicos e sedativos, contribuindo para o tratamento de inflamações, afecções genitourinárias, doenças respiratórias, dores reumáticas, e doenças no aparelho digestório.

A forma mais comum de usos dos produtos naturais é a infusão e a maceração, isto devido a grande quantidade de seus remédios serem preparados através da casca. Com essas plantas não foi diferente, porém o modo de preparo das plantas varia de acordo com as tradições culturais locais e/ou da parte utilizada segundo critérios regionais de indicações (De Paula et al., 2001). 


\subsection{Extratos vegetais: obtenção e características fitoquímicas}

Extratos são manipulações concentradas que podem estar na forma líquida, viscosa ou em pó e que são feitos a partir das partes secas da planta através de alguns métodos já predefinidos na literatura científica (Schulz et al., 2002). A extração ocorre através de processo físico-químico de transferência de massa, em que os sólidos solúveis e voláteis são extraídos por meio do contato entre o solvente e os sólidos (Clarke, 1985). As condições em que o processo de extração é realizado e a qualidade da matéria-prima vegetal influenciam o rendimento da extração (Wongkittipong et al., 2004; Schulz et al., 2002).

Esses processos de extração visam retirar os princípios ativos de uma determinada droga vegetal, através de um solvente obtendo-se, assim, formas terapêuticas para o manuseio e administração. Ferro (2008) descreve os processos de maceração, infusão, decoç̧ão, digestão, percolação e destilação como os principais métodos extrativos de propriedades medicinais e que são amplamente utilizados pela população (Tabela 3):

Tabela 3. Métodos extrativos utilizados para obtenção de extratos botânicos e consequente utilização das propriedades medicinais das plantas pela população.

\begin{tabular}{c|c}
\hline MÉTODO EXTRATIVO & DESCRIÇÃO \\
\hline Maceração & Contato da droga vegetal com o líquido por um período. \\
\hline Infusão & Adicionada água em ponto de ebulição à droga vegetal. \\
\hline Decocção & A água atinge o ponto de ebulição em contato com a droga vegetal. \\
\hline Digestão & O contato droga-solvente é mantido numa temperatura de 40 a 60 graus. \\
\hline Percolação & Ocorre a passagem do líquido extrator através de uma camada da droga \\
& vegetal moída. \\
\hline Destilação & A droga vegetal em contato com água ou álcool é submetida ao processo de \\
& destilação. \\
\hline
\end{tabular}

Adaptado de: Ferro (2002).

A escolha do solvente, a temperatura de extração e a ação mecânica (agitação e pressão) são fatores importantes que influenciam no resultado (Veggi, 2009). Além disso, a matriz vegetal apresenta uma microestrutura complexa formada por células, espaços intracelulares, capilares e poros. Dessa forma, a extração é influenciada pela estrutura molecular do soluto, o tamanho, localização e a ligação com outros componentes. Portanto, as características químicas do solvente, a estrutura e a composição diversificada do produto natural asseguram que cada sistema material-solvente mostra comportamento pelicular, o qual não pode ser previsto facilmente (Pinelo et al., 2004).

Em processos industriais, a fase inicial de preparação de um produto, exige a aplicação de uma técnica de extração do sólido-líquido para que possa isolar o material extraível contido nos mais variados tipos de matrizes vegetais. No entanto, avaliações de métodos extrativos que levam em consideração a eficiência e seletividade são fatores importantes para a extração de produtos naturais, pois a composição química das matrizes vegetais é bastante complexa ocorrendo a extração de vários tipos de compostos. Por isso, deve-se avaliar a eficiência dos métodos extrativos disponíveis (Simões et al., 2003).

Ainda a respeito da complexidade dos processos de extração de produtos naturais, Veggi (2006) relata que há uma variedade de compostos químicos que estão presentes em diferentes partes das plantas como folhas, raízes, flores, caules e frutos que têm capacidade de prover, além da nutrição básica, benefícios à saúde, como a prevenção e/ou tratamento de doenças. Por este motivo e pelo crescente interesse da população no consumo de alimentos, cosméticos e produtos medicinais, têm se intensificado o número de pesquisas utilizando derivados de fontes naturais no sentido não só de obter produtos 
derivados, que possam ter suas propriedades potencializadas, mas também de buscar alternativas para ampliar sua produção com o máximo de rendimento possível da planta.

Como já mencionado, a natureza do solvente e o processo de extração afetam o produto final (Tabela 4). Etanol, água ou a mistura de etanol e água são exemplos de solventes, dentre os mais utilizados, para produzir extratos líquidos. Mesmo com solventes idênticos, a própria técnica de extração pode resultar produtos com ações farmacológicas diferentes (Schulz et al., 2002). Dessa forma, a escolha do solvente é um fator importante no processo de extração sólido-líquido.

Tabela 4. Solventes descritos na literatura comumente utilizados para extração de componentes ativos.

\begin{tabular}{|c|c|c|c|c|c|c|}
\hline Metabólitos Solventes & Água & Etanol & Metanol & Clorofórmio & Éter & Acetona \\
\hline Ácidos Graxos & & & & & * & \\
\hline Alcaloides & & $*$ & $*$ & & * & \\
\hline Cumarinas & & & $*$ & & $*$ & \\
\hline Fenois & & & $*$ & & & $*$ \\
\hline Flavonoides & $*$ & $*$ & $*$ & $*$ & & $*$ \\
\hline Heterosídeos cardioativos & & & $*$ & & & \\
\hline Lignanas & & & $*$ & & & \\
\hline Polissacarídeos & $*$ & $*$ & $*$ & & & \\
\hline Quinonas & & & $*$ & & & \\
\hline Saponinas & $*$ & & $*$ & & & \\
\hline Taninos & $*$ & $*$ & $*$ & & & \\
\hline Terpenoides & $*$ & $*$ & $*$ & $*$ & $*$ & \\
\hline
\end{tabular}

Fonte: Modificado de Tiwari et al., (2011) e Cowan (1999).

O metabolismo vegetal é constituído por dois tipos de substâncias, os metabólitos primários e os secundários. O metabolismo primário é aquele que fornece as substâncias envolvidas nas funções básicas essenciais da vida celular. Os metabólitos secundários são específicos de cada espécie e participam das interações intra e intercelular do próprio organismo ou com células de outros organismos, também atuam em processos de polinização pela produção de substâncias que atraem os agentes vivos deste processo ou contribuem para a resistência dos organismos pela defesa contra pestes e outras doenças e estabelecendo a competência para a guerra química dos ajustes necessários à convivência e sobrevivência ambiental (BrazFilho, 2009). Os estudos fitoquímicos compreendem a extração e identificação dos constituintes presentes no vegetal, principalmente os metabólitos secundários que geralmente são os responsáveis por ações biológicas (Sonaglio et al., 2010). Os metabólitos secundários presentes nas plantas correspondem a uma série de substâncias com um perfil amplamente variado 
(Tabela 5), e muitos deles podem revelar efeitos biológicos quando utilizados pelo homem, tornando-se um importante foco de estudos para pesquisadores que visam o isolamento e a identificação dessas substâncias biologicamente ativas, potenciais candidatas para o desenvolvimento de novos fármacos (Kabera et al., 2014). Alguns agem como compostos de defesa contra herbívoros e patógenos, enquanto outros têm função como atrativos de polinizadores ou dispersores de frutos, na proteção contra a radiação ultravioleta, no suporte mecânico ou reduzindo o crescimento de plantas competidoras adjacentes (Santos, 2010; Taiz \& Zeiger, 2009).

Tabela 5. Propriedades terapêuticas dos metabólitos secundários estudados.

COMPOSTO PROPRIEDADE TERAPÊUTICA

Apresentam as propriedades de formar espuma abundante, associado a um poder hemolítico. Possuem

Saponinas atividade anti-inflamatória, analgésica, expectorante, antioxidante, redutora de colesterol, antiviral, antimicrobiana e antifúngica.

Flavonóides $\quad$ Possuem atividade antioxidante, anti-inflamatória, antitrombótica e vaso protetora.

Alcalóides Efeito antimuscarínico que está relacionado a sua ação antiespasmódica. A nível do sistema nervoso central podem exercer ação depressora (morfina, escopolamina), ou estimulante (esticnina, codeína)

Taninos Substâncias adstringentes e hemostáticas. Suas principais ações são: antisséptico, antidiarreico e cicatrizante.

Quinonas Indicados para pacientes que tem constipação para haver evacuação intestinal.

Cumarinas

Apresentam atividade anticoagulante, broncodilatador e efeito fotossensibilizante promotor de melanogênese sendo útil nos casos iniciais de vitiligo.

Fonte: Adaptado de Fonseca (2005); Monteiro (2005); Campanini, (2000); Meira Neto e Almeida (2015).

\section{Considerações Finais}

O uso de plantas medicinais está ligado a manifestações culturais e é uma forma de apresentar a importância que estas espécies têm em relação aos costumes de um povo. As famílias e espécies que predominaram nos estudos desenvolvidos com plantas da Caatinga, analisados na presente revisão, evidencia que a Caatinga possui alto potencial medicinal. Nesse levantamento, pode ser refletido esse elevado potencial medicinal, revelando a importância de estudos científicos mais acurados sobre as propriedades, métodos extrativos e a presença de metabólitos secundários.

No entanto, mais estudos envolvendo as plantas da Caatinga, são necessários para entender e validar a grande importância que essas famílias botânicas trazem as populações. Investigar potenciais antimicrobianos, anti-inflamatórios e neuroprotetor, por exemplo, podem possibilitar a descoberta de novas fontes de bioativos que irão contribuir para o desenvolvimento de novos medicamentos ou métodos de cura para as mais diversas patologias existentes. 


\section{Referências}

Agra, M. F., Silva, K. N, Basílio, I. J. L. D, Freitas \& P. F, Barbosa-Filho, J. M. (2008). Survey of medicinal plants used in the region Northeast of Brazil. Revista Brasileira de Farmacognosia 18: 472- 508.

Agra, M. F., Baracho, G. S, Nurit, K. Basílio, I. J. L. D. \& Coelho, V. P. M. (2007). Medicinal and poisonous diversity of the flora of “Cariri Paraibano", Brazil. Journal of Ethnopharmacology 111: 383-395.

Albuquerque, U. P. \& Lucena, R. F. P. (2004). Métodos e técnicas de pesquisa etnobotânica. 1 ed. Recife: Livro Rápido/NUPEEA. p.189.

Alves, J. J. A. (2007). Geoecologia da caatinga no semi-árido do Nordeste brasileiro. CLIMEP: Climatologia e Estudos da Paisagem, Rio Claro, v.2, n.1, p. 58-71.

Amorozo, M. C. M et al. (1996). A abordagem etnobotânica na pesquisa de plantas medicinais. In: DI STASI LC. (Org.). Plantas medicinais: Arte e Ciência, um guia de estudo interdisciplinar, 1a Ed, São Paulo: EDUSP, p.47-68.

Andrade, L. A.; Pereira, I. M.; Leite, U. T.; Barbosa, M. R. V. (2005) Análise da cobertura de duas fitofisionomias de Caatinga, com diferentes históricos de uso, no município de São João do Cariri, Estado da Paraíba. Cerne. v.11, n.3, p.253-262.

Angelo, P. M.; Jorge, N. (2007). Compostos fenólicos em alimentos - Uma breve revisão. Revista do Instituto Adolfo Lutz, Rio de Janeiro, v. 66, n. 1, p. 232240 .

Badke, M. R., Budó, M. L. D., Alvim, N. A. T., Zanetti, G. D. \& Heisler, E. E. (2012). Saberes e práticas populares de cuidado em saúde com o uso de plantas medicinais. Texto \& contexto enfermagem 21: 363-370.

Blanck, A. F., Fontes, S. M., Carvalho Filho, J. L. S., Alves, P. B., Silva, -Mann, R., Mendonça, M. C., Arrigoni -Blank, M. F. \& Rodrigues, M. O. (2005). Influência do horário de colheita e secagem de folhas no óleo essencial de melissa (Melissa officinalis L.) cultivada em dois ambientes. Revista Brasileira de Plantas Medicinais 8: 73-78.

BRASIL, Farmacopeia Brasileira. (2005). 4. ed. São Paulo: Atheneu.

Brum, T. F., Zadra, M., Froeder, A.L.F., Boligon, A. A., Frohlich, J. K. \& Athayde, M. L. (2011). Análise fitoquímica preliminar das folhas de Vitex megapotamica (Sprengel) Moldenke. Revista Saúde, Santa Maria 37: 57- 62.

Calixto, J. B., Scheidt, M., Otuki, M. \& Santos, A. R. (2001). Biological activity of plant extracts: novem analgesic drugs. Expert Opinion Emerging Drugs 2: 261- 279 .

Calixto, J. B. \& Siqueira Junior, J. M. (2008). Desenvolvimento de Medicamentos no Brasil: Desafios. Gazeta Médica da Bahia $78: 98-106$.

Castro, M. A. De; Bonilla, O. H.; Pantoja, L. D. M.; Mendes, R. M. S.; Edson-Chaves, B.; Lucena, E. M. P. Ethnobotanical knowledge of High School students about medicinal plants in Maranguape-Ceará. Research, Society and Development, [S. 1.], v. 10, n. 3, p. e8910313008, 2021. DOI: 10.33448/rsdv10i3.13008. https://rsdjournal.org/index.php/rsd/article/view/13008.

Camardelli, M., \& Napoli, M. F. (2012). Amphibian conservation in the Caatinga biome and semiarid region of Brazil. Herpetologica, 68: 31-47.

Cartaxo, S. L., Souza, M. M. A. \& Albuquerque, U. P. (2010). Medicinal plants with bioprospecting potential used in semi-arid northeastern Brazil. Journal of Ethnopharmacology 131: 326-342.

Carvalho, A. C. B., Nunes, D. S. G., Baratelli, T. G., Shuqair, N. S. M. \& Netto, E. M. (2007). Aspectos da legislação no controle dos medicamentos fitoterápicos. T\&C Amazônia 5: 26-32.

Carvalho, J. C. T. (2004). Fitoterápicos anti-inflamatórios: Aspectos químicos, farmacológicos e aplicações terapêuticas. São Paulo: Tecmedd 480 p.

Clarke, N. A. (1985). Surface memory effects in liquid crystals: Influence of surface composition. Physical Review Letter 55: 292 - 295.

Costa, A. F. (1994). Farmacognosia (Vol. 3, 5a ed). Lisboa: Fundação Calouste Gulbenkian.

Cruz, R. R. P.; Freire, A. I.; Pereira, A. M.; Ribeiro, R. P.; Alves, M. M. Momordica charantia L. in the treatment of diabetes melittus. Research, Society and Development, [S. 1.], v. 9, n. 7, p. e15973769, 2020. DOI: 10.33448/rsd-v9i7.3769. https://rsdjournal.org/index.php/rsd/article/view/3769.

Cowan, M. M. (1999). Plant products as antimicrobial agents. Clinical microbiology reviews 12: 564-582.

Das, K., Tiwari, R. K. S. \& Shrivastada D. K. (2010). Techniques for evaluation of medicinal plant products as antimicrobial agent: Current methods and future trends. Journal of Medicinal Plants Research 4: 104-111.

Lima, S. G., Citó, A. M. G., Lopes, J. A. D., Neto, J. M. M., Chaves, M. H. \& Silveira, E. R. (2010). Fixed and volatile constituents of genus Croton plants: C. Adenocalyx baill - Euphorbiaceae. Rev. Latinoam. Quim 38:133-144.

Drumond, M. A. (2013). Potencialidades de algumas espécies arbóreas madeireiras do bioma Caatinga. In: SILVA, Márcia Vanusa et al. (Org.) A Caatinga e seu potencial. 23 ed. Recife: Ed. Universitária 1: 1-18.

Estrela, C. (2018). Metodologia Científica: Ciência, Ensino, Pesquisa. Editora Artes Médicas.

Fernandes, A. G., Alencar, J. W. \& Matos, F. J. A. (1971). Canelas silvestres nordestinas: aspectos botânicos, químicos e farmacológicos. Ciência e Cultura $32: 26-33$. 
Fernandes, P. R. D.; Bizerra, A. M. C. Quantitative evaluation of antioxidant activities of native plants of the Alto Oeste Potiguar Region / RN. Research, Society and Development, [S. 1.], v. 9, n. 1, p. e48911578, 2020. DOI: 10.33448/rsd-v9i1.1578. https://rsdjournal.org/index.php/rsd/article/view/1578.

Ferro, Degmar. (2008). Fitoterapia: conceitos clínicos. Atheneu.

Figueiredo, M. A., Rodal, M. J, N., Barbosa, M. R. V. \& Harley, R. M. (2004). Diagnóstico da vegetação nativa do bioma Caatinga. Pg. 48-90 in J. M. C. Silva, M. Tabarelli, M. Fonseca, \& L. Lins, editors. Biodiversidade da Caatinga: áreas e ações prioritárias para a conservação. Ministério do Meio Ambiente, Brasília.

Franco E. A. P. \& Barros, F. R. M. (2006). Uso e diversidade de plantas medicinais no Quilombo Olho D’água dos Pires, Esperantina, Piauí. Rev. Bras. Pl. Med 8:78-88.

García, A. Á. \& Carril, E.P. (2009). Metabolismo secundário de plantas. Reduca (Biologia) Serie Fisiologia vegetal 2:119-145.

Gibaja, S. (1998). Pigmentos naturales quinónicos. 1 ed. Lima, UNMSM, Fondo editorial, 277 p.

Giulietti, A. M., Bocage Neta, A. L., Castro, A. A. J. F., Gamarra-Rojas, C. F. L., Sampaio, E. V. S. B., Virgínio, J. F., Queiroz, L. P. \& Gomes, A. P. S. (2006). Revisão das espécies sulamericanas de Croton L. subgen. Croton sect. Argyroglossum Baill. (Crotonoideae- Euphorbiaceae). 124p. Tese de Doutorado. Universidade Federal Rural de Pernambuco, Recife.

Gomes, E. C. S., Barbosa, J., Vilar, F. C. R., Perez, J. O., Vilar, R. C.; Freire, J. L. O., De Lima, A. N., Dias, T. J. (2008). Plantas da caatinga de uso terapêutico: levantamento etnobotânico. Engenharia Ambiental: Pesquisa e Tecnologia, 5: 74-85.

Govaerts, R., Frodin, D.G. \& Radliffe-Smith, A. (2000). World Checklist and Bibliography of Euphorbiaceae (and Pandaceae). The Board of Trustees of the Royal Botanic Gardens, Kew 1-4:1-1622.

Guedes, T. B., Sawayra, R. J., \& C Nogueira, C. (2014). Biogeography, vicariance and conservation of snakes of the neglected and endangered Caatinga region, northeastern Brazil. Journal of Biogeography 41:919-931.

Harbore, J. B. (1998). Phytochemial methods: a guide to modern techniques of plan analysis. Chapman and Hall. 3.ed.

Helfand, W. H. \& Cowen, D.L. (1990). Pharmacyan illustrated history. New York: Harry N. Abrams.

Kabera, J. N., Semana, E., Mussa, A. R. \& He, X. (2014). Plant secondary metabolites: biosynthesis, classification, function and pharmacological properties. Journal of Pharmacy and Pharmacology 2:377-392.

Koche, J. C. (2011). Fundamentos de metodologia científica. Petrópolis: Vozes. http://www.brunovivas.com/wpcontent/uploads/sites/10/2018/07/K\%C3\%B6che-Jos\%C3\%A9-Carlos0D0AFundamentos-de-metodologia-cient\%C3\%ADfica-_teoriada0D0Aci\%C3\%AAncia-e-inicia\%C3\%A7\%C3\%A3o-\%C3\%A0-

pesquisa.pdfhttps://repositorio.ufsm.br/bitstream/handle/1/15824/Lic_Computacao_Metodologia-Pesquisa-Cientifica.pdf?sequence=1

Leal, I. R., Da Silva, J., Cardoso, M., Tabarelli, M. \& Lacher, ACHER, T. E. (2005). Changing the course of biodiversity conservation in the Caatinga of northeastern Brazil. Conservation Biology 19:701-706.

Lima, L. R. \& Pirani, J.R. (2008). Revisão taxonômica de Croton sect. Lamprocroton (Müll. Arg.) Pax (Euphorbiaceae s.s). Biota Neotrópica 8: 177-231

Lopes, C. R. et al. (2005). Folhas de chá. Viçosa: UFV.

Lorenzi, H. \& Matos, F. J. A. (2008). Plantas medicinais no Brasil: nativas e exóticas. São Paulo: Instituto Plantarum.

Ludke, M. \& Andre, M. E. D. A. (2013). Pesquisas em educação: uma abordagem qualitativa. São Paulo: E.P.U.

Maciel, M. A. M., Pinto, A. C. \& Veiga Júnior, V.F. (2002). Plantas Medicinais, a necessidade de estudos multidisciplinares. Quimica Nova 25: 429-438.

Martins, A. P., et al. (2000). Antimicrobial Activity and Chemical Composition of the Bark Oil of Croton stellulifer, an Endemic Species from S. Tome e Principe. Planta Med. 66: 647-650.

Martins, E. R., Castro, D. M., Catellane, D. C. \& Dias, J. E. (2000). Plantas Medicinais. Editora Universidade/UFV, Vicosa.

Matos, F. J. A. (1997). Introdução à fitoquímica experimental. (2a. ed.) Fortaleza: EUFC.

Matos, F. J. A. (1999). Plantas da medicina popular do Nordeste: propriedades atribuídas e confirmadas. Fortaleza: EDUFC.

MMA (Ministério do Meio Ambiente). (2004). Biodiversidade da Caatinga: áreas e ações prioritárias para conservação. Secretaria de Biodiversidade e Florestas. MMA, Brasília.

Mogensen, A. O. (1982). Choice of solvent in extraction, In: AIChEMI modular instructions: Series B, Stagewise and mass transfer operations. New York: American Institute of Chemical Engineers (Extraction and Leaching 3. Module B3.5).

Monte, F. J. Q. (1980). Contribuição ao conhecimento químico de plantas do Nordeste - Croton argyrophylloides Muell. Arg. Fortaleza, 1980. Dissertação (Mestrado Acadêmico em Química Orgânica) - Universidade Federal do Ceará.

Moreas, M. E. A. \& Santana, G. S. M. (2001). Aroeira-do-sertão: um candidato promissor para o tratamento de úlceras gástricas. Funcap 3: 5-6.

Morais- Costa, M. S., Carvalho,O., Cardoso- Filho, B. C., Ferreira. S., Carvalho, W. A. \& Silva, D. A. (2009). Riscos de intoxicação de animais na pecuária, por plantas tóxicas: uma revisão bibliográfica. Anais do IX Congresso de Ecologia do Brasil, 13 a 17 de Setembro de 2009 , São Lourenço- MG 
Moreira, J. N., Lira, M. A., Santos, M. V. F., Ferreira, M. A., Araújo, G. G. L., Ferreira, R. L. C. \& Silva, G. C. (2006). Caracterização da vegetação de caatinga e da dieta de novilhos no Sertão de Pernambuco. Pesquisa Agropecuária Brasileira 41: 1643-1651.

Mouco, G., Bernardino, M. J., Cornélio, M. L. (2003). Controle de qualidade de ervas medicinais. Revista biotecnologia Ciência e Desenvolvimento 31:68-73.

Ncube, N. S., Afolayan, A. J. \& Okoh, A. I. (2008). Assessment techniques of antimicrobial properties of natura compounds of plant origin: current methods and future trends. African Journal of Biotechnology 7: 1797-1806.

Oliveira V. B, Zuchetto M, Oliveira C. F, Paula C. S, Duarte A. F. S, Miguel M. D. \& Miguel O. G. (2016). Efeito de diferentes técnicas extrativas no rendimento, atividade antioxidante, doseamentos totais e no perfil por CLAE-DAD de Dicksonia sellowiana (presl.). Hook, dicksoniaceae. Rev Bras Plantas Med 18:230-239.

Payo, H. A., Dominicis, M. E., Mayor, J., Oquendo, M. \& Sarduy, R. (2001). Tamizaje fitoquímico preliminar de espécies del género Croton L. Revista Cubana de Farmacia. 2001.

Pereira A. S. et al. (2018). Metodologia da pesquisa científica. [free e-book]. Santa Maria/RS. Ed. UAB/NTE/UFSM.

Pinelo, M., Rubilar, M., Sineiro, J. \& Núñez, J. (2004). Extraction of antioxidant phenolics from almond hulls (Prunus amygdalus) and pine sawdust (Pinus pinaster). Food Chemistry 85: 267-273.

Poirot, R., Prat, L., Gordon, G., Diard, C., Autret, J. M. (2006). Fast Batch to Continuous Solid-Liquid Extraction from Plants in Continuous Industrial Extractor. Chemical \&. Engineering Technology 29: 46-51.

Rosa, C., Câmara, S. G. \& Béria, J. U. (2011). Representações e intenção de uso da fitoterapia na atenção básica à saúde. Ciências \& Saúde Coletiva 16: 311 318.

Santana, J. A. S. \& Souto, J. S. (2006). Diversidade e estrutura fitossociológica da caatinga na estação ecológica do Seridó, RN. Revista de Biologia e Ciência da Terra 6:232-242.

Schenkel, E. P., Gosmann, G., Mello, J. C. P., Mentz, L. A., Petrovick, P. R. Farmacognosia, da planta ao medicamento. 6. ed. Porto Alegre/Florianópolis: UFRS, cap. 13: 290- 326.

Schulz, V., Hansel, R. \& Tyler, V. (2002). Fitoterapia Racional: Um guia de fitoterapia para as ciências da saúde. São Paulo: Manole, 2002. 386 p.

Shale, T. L., Stirk, W. A. \& Van Staden, J. (1999). Screening of medicinal plants used in Lesotho for anti-bacterial and anti-inflammatory activity. Journal of Ethnopharmacology 67: 347-354.

Silva, A. I., Sá-Filho, G., Oliveira, L., Guzen, F., Cavalcanti, J., \& Cavalcante, J. (2021). Perfil fitoquímico de extratos etanólicos e metanólicos do Croton blanchetianus. Revista Brasileira Multidisciplinar, 24(1), 134-142. https://doi.org/10.25061/2527-2675/ReBraM/2021.v24i1.1057

Silva, L. N., Trentin, D. S., Zimmer, K. R., Treter, J., Brandelli, C. L. C., Frasson, A. P., Tasca, T., Silva, A. G., Silva, M. V. \& Macedo, A. J. (2015). Antiinfective effects of Brazilian Caatinga plants against pathogenic bacterial biofilm formation. Pharmaceutical Biology, 53 :464-468.

Silva, N. L. A., Miranda, F. A. A. \& Conceição, G. M. (2010). Triagem Fitoquímica de Plantas de Cerrado, da Área de Proteção Ambiental Municipal do Inhamum, Caxias, Maranhão. Scientia Plena 6: 1-17.

Silva, T. R. G., Martins., T. D. T., Silva, J. H. V., Silva, L. P. G., Pascoal, L. A. F., Oliveira, E. R. A. \& Brito, M. S. (2012). Inclusão de óleos essenciais como alimentos fitoterápicos na dieta de suínos, Revista Brasileira de Saúde e produção animal 13:181-191.

Simões, C.M.O. (2010). Farmacognosia: da planta ao medicamento. $6^{\mathrm{a}}$ ed. Porto Alegre: UFRGS. ISBN: 978-85-7025-927-1.

Simões, C. M. O., Schenkel, E. P., Gosmann, G., Mello, J. C. P., Mentz, L. A. \& Petrovick,P. R. (2004). Farmacognosia: da planta ao medicamento. 5. ed. Porto Alegre.

SNIF FLORESTAL. (2021). http://snif.florestal.gov.br/pt-br/perda-da-cobertura-florestal/262-mapas

Sonaglio, D; Ortega, G. \& Petrovick, P. R. \& Bassani, V. L. (2003). Desenvolvimento tecnológico e produção de fitoterápicos. In: Simões, C. M. O., Schenkel, E. P., Gosmann, G., Mello, J. C. P., Mentz, L. A. \& Petrovick,P. R. (org.) Farmacognosia: da planta ao medicamento. 5. ed. rev. e ampl.,Porto Alegre: UFRGS, Florianópolis: UFSC. cap. 13, p. 289-32

Taiz, L. \& Zeiger, E. (2009). Fisiologia vegetal. 4. ed. Porto Alegre: Armed, 849p.

Tiwari, P., Kumar, B., Kaur, M., Kaur, G. \& Kaur, H. (2011). Phytochemical screening and extraction: a review. Intern Pharm Science 1:98-106.

Trovão, D. M. et al. (2007). Variações sazonais de aspectos fisiológicos de espécies da Caatinga. R. Bras. Eng. Agríc. Ambiental 11: $307-311$.

Vanzolini, P. E. (1976). On the lizards of a Cerrado-Caatinga contact: evolutionary and zoogeographical implications (Sauria). Papéis Avulsos de Zoologia, São Paulo, 29, 111-119.

Veggi, Priscilla Carvalho. (2006). Obtenção de extratos vegetais por diferentes métodos de extração: estudo experimental e simulação dos processos. $165 \mathrm{f}$. Dissertação (Mestrado) - Curso de Engenheira de Alimentos, Universidade Estadual de Campinas, Campinas.

Velloso, A. L., Sampaio, E. V. S. B. \& Pareyn, F. G. C. (2002). Ecorregiões propostas para o bioma caatinga. Recife: Associação Plantas do Nordeste; Instituto de Conservação Ambiental, The Nature Conservancy do Brasil.

Verpoorte, R. (1998). Exploration of nature's chemodiversity: the role of secondary metabolites as leads in drug development. Drug Discovery Today 3: 232238. 
Research, Society and Development, v. 10, n. 13, e140101321096, 2021

(CC BY 4.0) | ISSN 2525-3409 | DOI: http://dx.doi.org/10.33448/rsd-v10i13.21096

Wina, E., Muetzel, S. \& Becker,K. (2005). The Impact of Saponins or SaponinContaining Plant Materials on Ruminant Production - A Review. Journal of Agricultural and Food Chemistry 53: 8093-8105.

Wongkittipong, R., Prat, L., Damronglerd, S. \& Gourdon, (2004). C. Solid-liquid extraction of andrographolide from plants-experimental study, kinetic reaction and model. Separation and Purification Technology 40: 147-154.

Yadav, R., \& Agarwala, M. (2011). Phytochemical analysis of some medicinal plants. Journal of phytology 3:10-4. 